\title{
Control problems in quantum systems
}

\author{
WU ReBing ${ }^{1,2 *}$, ZHANG Jing ${ }^{1,2}$, LI ChunWen ${ }^{1,2}$, LONG GuiLu $^{2,3}$ \& TARN TzyhJong ${ }^{2,4}$ \\ ${ }^{1}$ Department of Automation, Tsinghua University, Beijing 100084, China; \\ ${ }^{2}$ Center for Quantum Information Science and Technology, TNList, Beijing 100084, China; \\ ${ }^{3}$ Department of Physics, Tsinghua University, Beijing 100084, China; \\ ${ }^{4}$ Electrical and Systems Engineering Department, Washington University in St. Louis, St. Louis, Missouri 63130, USA
}

Received October 23, 2011; accepted December 29, 2011; published online April 24, 2012

The past decade or two has witnessed tremendous progress in theory and practice of quantum control technologies. Bridging different scientific disciplines ranging from fundamental particle physics to nanotechnology, the goal of quantum control has been to develop effective and efficient tools for common analysis and design, but more importantly would pave the way for future technological applications. This article briefly reviews basic quantum control theory from the perspective of modeling, analysis and design, as well as considers future research directions.

quantum control, control theory, quantum physics

Citation: Wu R B, Zhang J, Li C W, et al. Control problems in quantum systems. Chin Sci Bull, 2012, 57: 2194-2199, doi: 10.1007/s11434-012-5193-0

Quantum control refers to the design of control strategies in systems that obey the principles of quantum mechanics, e.g. microscopic systems with few atoms or photons that were addressed early in the lecture "Plenty of Room at the Bottom" given by Richard Feynman in 1959 [1]. This dream has been pursued since 1960s right after the invention of lasers, which illuminated the hope to coherently control chemical reaction processes, but it was not until the end of 20th century when a burst of successes occurred in controlling ultrafast quantum dynamics. Nowadays, quantum control protocols are being introduced to more fields such as quantum information processing [2] and nanomaterials and nanodevices [3].

The inherent power afforded quantum control results from the unique nonclassical features of quantum mechanics. Essentially, orthogonal eigenstates of a quantum observable (corresponding classically identifiable states) can form superpositions and thereby span a much larger space of quantum states available for coherent manipulation; quantum tunneling allows crossing of energy barriers without as much work as in classical mechanics, thus potentially improve sensitivity and controllability. However, there is no free lunch

*Corresponding author (email: rbwu@ tsinghua.edu.cn) on the other side; these advantages can only be exploited under extreme spatial (atomic or subatomic) and temporal (femtosecond and attosecond) physical scales. To realize the power of quantum control, vulnerable coherence and entanglement in quantum states need to be protected and measured before being destroyed by intermediate interacting environments. The earliest quantum control studies from the $1960 \mathrm{~s}$ to the 1980s focused on (macroscopic) quantum ensembles in plasmas and laser devices, nuclear accelerators, and nuclear power plants [4] (mostly in former Soviet Union), in which the systems were modeled as quantum harmonic oscillators, which have little difference with those from classical systems [5,6]. In the 1980s, Tarn's group completed a series of studies on general (linear or nonlinear) quantum systems in regard to modeling [7], controllability [8], invertibility [9], and quantum nondemolition filter problems [10]. In Europe, Belavkin's investigations [11-13] on the optimal estimation of quantum signals in quantum channels shows that quantum feedback control and optimal control is in principle feasible using quantum filters and nondemolition measurements.

Photonic control of quantum chemical processes might be the most productive area to date [14-18]. After discovering the IVR (intramolecular vibrational resonance) phenomenon 
that hinders the control of molecular dynamics, investigators realized that vibrational (electronic) molecular dynamics can only be controlled at femtosecond (attosecond) scales. Only at such intervals can the dominant degrees of molecular freedom be manipulated against IVR via coherent constructive and destructive superpositions. Early single-parameter control schemes include the Brumer-Shapiro phase control scheme [19,20] and Tannor-Rice time-delay scheme [21]. Later on, optimal control theory (OCT) was applied to molecular spectroscopy $[22,23]$ as well as more complex cluster systems [24]. In actual system implementations, the introduction of model-independent learning algorithms [25] led to successful breakthroughs in the laboratory $[18,26]$. By 2010, over 150 experiments have been successfully carried out in the laboratory, and a much greater number of numerical simulations have been reported [27]. In the AMO 2010 report composed by National Research Council of United States [28], it was pointed out that the future development of quantum control will challenge extremely critical physical circumstances including (1) systems in microscopic scales (e.g. nanoscale); (2) extremely low temperatures; (3) ultrafast dynamics; (4) high-energy limits; (5) information theoretic limits. Fields touching on any of these exciting physics will open up numerous opportunities for interdisciplinary research.

The field of quantum control itself is evolving rapidly. Figure 1 shows the statistics of research articles that were published over the past twenty years (data obtained from Web of Science, Thomson Reuters Co.). It can be seen that past quantum control studies were mostly in the areas of physics and chemistry, but in recent years research from engineering fields has grown very quickly. Moreover, several monographs have been published on quantum control with authors coming from chemistry, quantum optics and control theory

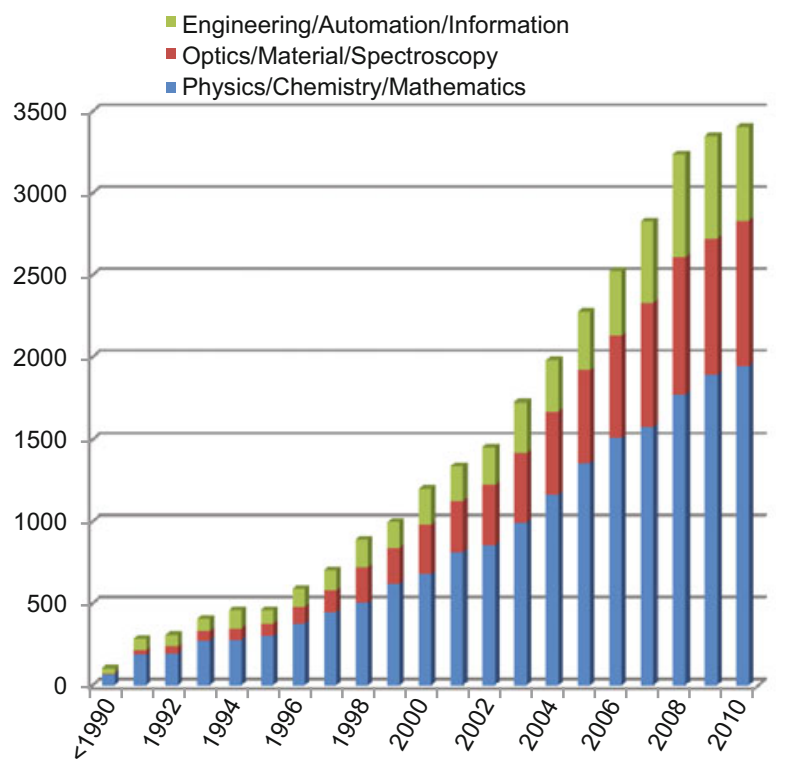

Figure 1 Statistics of the number of SCI publications on quantum control in the science and engineering fields (Source: Web of Science).
$[14,15,29,30]$ and the interested reader can learn more about the state-of-art in quantum control from various review articles [2, 27,31]. More recently, interdisciplinary studies on quantum control have also attract much attention in China [32-37]. In this review, we aim to give a general review over the fundamental problems and theory from the viewpoint of system control. In Section 1, we will describe how to model quantum control system under semiclassical controls. Then, in Section 2, we discuss the fundamental analysis problems of quantum control including stability and controllability. In Section 3, control design methods will be introduced, particularly on optimal control design methods. Finally, perspectives will be presented.

\section{Modeling of quantum control systems}

Physical objects in nature are usually classified as either matter or fields; thus the goal of quantum control can likewise be classified as the control of matter and the control of fields. The control of matter employs well-controlled light sources to effectively manipulate the dynamics of matter (e.g. molecules, spins, electrons or macroscopic subjects such as quantum dots and superconducting devices). The control of field refers to the preparation of optical states (e.g. singlephotons or squeezed states) for high-precision measurements or information processing, or the generation of new light sources (of high frequency or high intensity) for more advanced control or measurement of matter [38], such as via higher-order harmonic generation (HHG) [39]. To control matter or fields, the object needs to interact with the control, in which the interaction mechanism of matter or field plays the central role. In practice, coherent (laser) fields are often employed to manipulated quantum dynamics. If the photon number contained in the field is large $\left(\sim 10^{5} / \mathrm{cm}^{3}\right.$ in each mode), the field can be approximated with high precision by a classical electromagnetic field, called the semiclassical approximation. Moreover, if the size of matter being radiated is far smaller than the wavelength of light, the interaction with the field depends only on the position $\boldsymbol{r}_{0}$ of the center of mass. Under an appropriately chosen gauge, the interaction Hamiltonian can be written as

$$
H_{\text {int }}=\boldsymbol{E}\left(\boldsymbol{r}_{0}, t\right) \cdot \boldsymbol{D},
$$

where $\boldsymbol{D}$ is the dipole operator of the quantum system. If the size of matter is comparable with the wavelength, a coupled Maxwell equation representing the evolution of the field will be required. Although no direct interaction exists between electromagnetic fields, field control can be indirectly done by another external field via some linear or nonlinear medium.

Under appropriate physical circumstances, a semiclassical control system over a time-varying quantum wave function $\Psi(t)$ can be expressed in the following general form:

$$
i \hbar \frac{\partial \Psi(t)}{\partial t}=\left\{H_{0}+\sum_{k=1}^{r} u_{k}(t) H_{k}\right\} \Psi(t),
$$


where $H_{0}$ is the internal Hamiltonian and $H_{k}(k=1, \ldots r)$ are control Hamiltonians activated by associated time-dependent control parameters $u_{k}(t)$ that can be tuned by their frequency, phases, or profile. Here the system can be physically either matter or field. Such a system is referred to as a bilinear control system because the equation is linear in both the state and the control functions. From the view of system control theory, it is also convenient to define a target to evaluate the control performance. For example, a natural choice is the expectation value of some system observable

$$
y(t)=\langle\Psi(t)|O| \Psi(t)\rangle,
$$

which can be measured when the system is a quantum ensemble or the system is measured for a sufficient number of times.

In quantum information science, the evolution operator itself is often a target of control, which leads to the following form of control system:

$$
i \hbar \frac{\partial U(t)}{\partial t}=\left\{H_{0}+\sum_{k=1}^{r} u_{k}(t) H_{k}\right\} U(t),
$$

where the unitary evolution operator $U(t)$ always starts from the identity operator. The corresponding output is often chosen as the fidelity with some desired quantum gate, i.e. $y=\left|\operatorname{Tr}\left(W^{\dagger} U(T)\right)\right|$, where $W$ is the target unitary transformation. In practice, the system is always under the influence of external environments and should be modeled as an open quantum system, but we will not go further into this aspect. Interested readers can refer to the literature [30].

In recent years, networked quantum systems drew much attention, which is crucial in building quantum structures and thus enabling construction of large-scale electronic circuits at the quantum level. More importantly, as the core of control theory, the implementation of feedback control relies on the possibility of integrating quantum components into feedback loops. In classical system theory [40], it is natural to treat each component of a composite system as an objective with inputs and outputs:

$$
\dot{x}(t)=f[x(t), u(t)], \quad y(t)=g[x(t), u(t)],
$$

where the input $u(t)$ causally affects the evolution of the state $x(t)$ through the dynamical equation, which subsequently influences the variation of the output $y(t)$ as a function of the state and control variables. However, this general structure needs to be carefully treated in quantum systems when the input and/or output are quantum variables, as they acts on different Hilbert spaces. The seminal paper [41] presented the Langevin (as well as master) equations for quantum systems for which the input and output are optical fields:

$$
\dot{a}=-i \omega a-\frac{\gamma}{2}+\sqrt{\gamma} b_{\text {in }}, \quad b_{\text {out }}=b_{\text {in }}+\sqrt{\gamma} a .
$$

Here the input and output act on the same Hilbert space, representing the incoming and outcoming free-space fields.
Based on the theory, systematic studies have been performed on linear quantum networks involving concatenation, series product, and parallel product, which are interesting topics that are quite different from classical control systems. In the space of coherent states, a beautiful parallel with classical harmonic oscillator networks can be observed [42], although more interesting are systems having nonlinear Hamiltonians at the single-photon level. For more details, readers may look into another paper written by Zhang and James on the topic of coherent feedback control.

\section{System analysis: Stability and controllability}

A major motivation of system control theory is to achieve high control performance under limited resources and uncontrollable disturbances. However, a more fundamental requirement is to maintain the system at a desired working status; this calls for stability. The concern for stability in quantum system dates back to the very beginning of quantum physics when the answer for "why matter stays as it is?" was pursued [43]. For example, an atom consisting of a nucleus surrounded by electrons cannot exist stably in classical electrodynamics theory because electrons will eventually fall to the surface of the nucleus after losing energy via electromagnetic radiation. It can only be explained in quantum theory by the lower-bound eigenstructure of electronic states.

During the past century, quantum stability was mainly studied by mathematical physicists. To engineer quantum systems, it is also of practical importance to keep quantum systems stable under open-loop or close-loop designs. A typical circumstance is when a system is desired to be stabilized on (or as close as possible to) the unit sphere of pure states against decoherence effects. To our knowledge, a quantum stability study that should be physically meaningful and convenient for engineering control design seems to be lacking. For open-loop control systems, a frequently discussed problem is the stability of systems under periodic [44] or chaotic [45] external fields, where the system is stable if the kinematic energy is bounded. For feedback control purposes, the concept of input-output stability is extended to quantum control systems [46]. There are still many open problems in parallel with various stability concepts in classical theory, e.g. input-output stability, input-to-state stability, stochastic stability and structural stability.

As another fundamental concept in control theory, controllability refers to the ability of steering quantum systems between arbitrary states. The controllability issue was first attacked by Tarn et al. in the early 1980s with respect to quantum systems with finite-dimensional controllability Lie algebras; later this was extended to time-dependent systems [47] and systems with infinite-dimensional controllability Lie algebras [48]. A special class of such systems was also extensively studied in the control of molecular systems, where quantum systems were assumed to possess at first approx- 
imation a finite number of levels. Based on Lie algebraic analysis, the controllability of such systems received careful study in regard to the dipole structure [49]. Recently, an interesting field-atom system was studied [50] via methods established in differential geometry, showing that a two-level system interacted with a single optical mode is controllable over any finite-dimensional subspace without the need for the rotating-wave approximation, which was thought to be crucial in understanding the dynamics of strongly interacting quantum systems.

To summarize, the study of controllability for finite-level quantum systems has been relatively complete, although that of infinite-level systems, especially systems with unbounded and continuous spectra, is extremely difficult. Moreover, it appears that controllability is only of interest to control theoreticians and mathematicians, whereas no direct relevance can be seen to actual control design. However, this is not the case [51], as controllability determines the complexity of finding optimal controls (i.e. search efforts). The more a system is controllable, the easier is the search to reach a global optimal solution. Thus, delving further into the fundamental study of controllability is deservedly worthwhile.

\section{Control design methodologies}

The design methods of quantum control can be roughly classified as open-loop and feedback methods. The open-loop design (see Figure 2 for schematics) determines the control before applying the control onto the system [27], while feedback design (see Figure 3 for schematics) adaptively adjusts the system dynamics during the evolution according to information acquired from the system [31]. The body of literature on open-loop control is much larger than that of feedback control, in contrast to classical control systems where feedback control is dominant. This is because the measurement required for feedback is difficult; one can even do it, though the trade-off between inevitable back-action and the inquired information will have to be considered. It is not even known whether feedback will eventually improve the control performances. Only recently has feedback attracted any attention.

Here we will review the main design methods in openloop control design, and the closed-loop control methods will be surveyed by Zhang and James in this special topic. More specifically, we will focus on optimal control methods, which cover most of the relevant literature. There are also design methods based on physical intuition (e.g. [52,53]) and Lie algebraic decompositions (e.g. [54]), but we will not discuss these in any detail. Every control target can be described by one or multiple costs as functional(s) of the control fields, say $J[u(\cdot)]$. For example, the frequently-used expectation

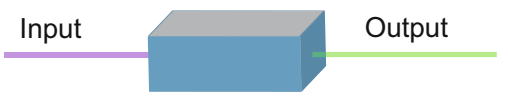

Figure 2 Schematics for quantum open-loop control system.

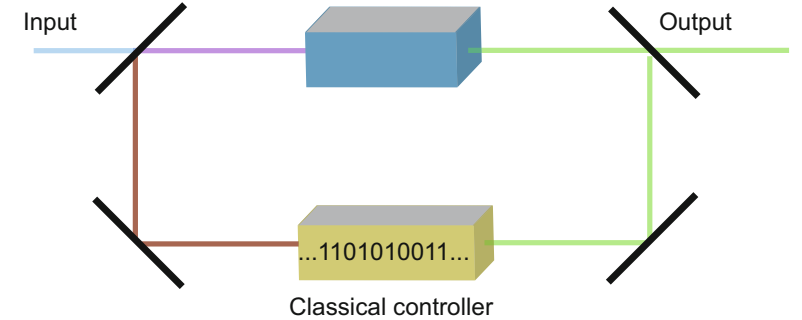

(a)

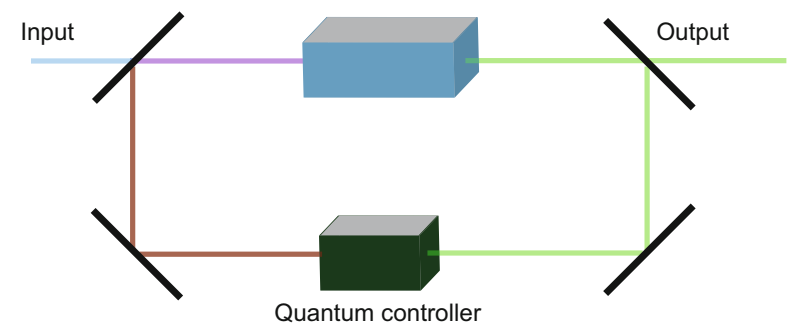

(b)

Figure 3 Statistics from quantum feedback control system. (a) Quantum measurements record information about a system as classical signals that are then processed to update control inputs. (b) A so-called coherent feedback loop where the system output is directly processed by a quantum controller instead of being measured.

value of some observable is an implicit functional of control, because the state depends on the control through the Schrödinger equation. In practice, one can also choose cost functionals as the state transition probability [53], gate fidelity [55], decoherence suppression [56], fluence minimization [57], and the time expended in control [58].

The more prevalent optimization algorithms used in quantum control problems can be classified as iterative-type [55, 59] and gradient-type [60-62] algorithms. All these algorithms start from an initial guess of the control field that can be randomly chosen or come from the natural form of laboratory implementations. The iterative-type algorithms generally converge quickly although their convergence is heavily dependent on the initial guess; the gradient-type algorithms are more stable as long as the step-size is sufficiently small, but the drawback is that their convergence is very slow, especially near optimal solutions. In addition, a common requirement is that system Hamiltonians have to be precisely known, which is almost impossible in laboratory situations. Even when the Hamiltonian is available, the numerical integration of the time-dependent Schrödinger equation will usually be very heavy. To overcome such difficulties in laboratory, evolutionary-type algorithms $[25,63]$ have been proposed in recognition of the fact that the most difficult part (solving Schrödinger equation) can actually be done by nature herself, and what the algorithm needs to do is only to measure the output and to adjust the control according to the designed algorithm. In this way, one can also tune his control variables without knowing exactly what happens to the system dynamics. Theoretically, such algorithms are less efficient than Hamiltonian-dependent algorithms, but in prac- 
tice it can evolve very rapidly without having to solve the Schrödinger equation.

Both types of algorithms can suffer from the possible existence of so-called false traps, i.e. the search could converge to a control solution that is locally but not globally optimal. Such problems are generally more severe if optimization resources are limited, which appears to be the case for quantum control in the laboratory. However, it is surprising [64] that most of the reported simulation results observed quick convergence to global optimal solutions, and experiment yields were most remarkably improved, implying that the traps are not supposed to be ubiquitous in quantum control. This observation was supported via mathematical analysis of the socalled quantum control landscape [64]. It was proved that if the system is controllable, then upon some mild assumptions there exist no traps on the landscape in maximizing observable expectation values [65], gate fidelity [66], and even the costs in open systems [67]. This opens up a new field in which investigators can obtain a deeper understanding of quantum control and optimization, and with such understanding it is possible to develop more efficient quantum control algorithms.

\section{Perspectives}

Quantum control is a new interdisciplinary field requiring collaborative research performed by researchers from different disciplines; such collaborations have already come into being. From chemistry to NMR, superconducting devices, and quantum dots, quantum control has shown itself powerful in many experimental setups. Although, in the past, quantum physics has been seen to be too hard for engineers to understand, and engineering methodologies have been only accepted reluctantly by physicists, the gaps in both has been greatly reduced during the past decades as experimental technologies have quickly develop. Motivated by explorations of the ultimate physical limits and the great impact on future technologies, the fusion of theory and practice in regard to quantum control will open up tremendous research opportunities.

This work was supported by the National Natural Science Foundation of China (60904034, 61174084 and 61134008) and Tsinghua National Laboratory for Information Science and Technology (TNList) Cross-discipline Foundation.

1 Feynman R. There's plenty of room at the bottom. Annual Meeting of the American Physical Society, California Institure of Technology, Pasadena, CA, 1959

2 Vandersypen L M, Chuang I L. NMR techniques for quantum control and computation. Rev Mod Phys, 2004, 76: 1037-1069

3 Grigorenko I, Rabitz H. Optimal control of the local electromagnetic response of nanostructured materials: Optimal detectors and quantum disguises. Appl Phys Lett, 2009, 94: 253107

4 Butkovskiy A, Samoilenko Y I. Control of quantum-mechanical processes and systems. In: Mathematics and its Applications. Boston:
Kluwer Academic Publishers, 1990

5 Butkovskii A, Pustyl'nikova E. Controlling the coherent states of a quantum oscillator. Automation Remote Contr, 1982, 43: 1393-1398

6 Butkovskii A, Pustyl'nikova E. Control of coherent states of quantum systems with a quadratic hamiltonian. Automation and Remote Control, 1984, 45: 1000-1008

7 Tarn T J, Huang G M, Clark J W. Modelling of quantum mechanical control systems. Math Model, 1980, 1: 109-121

8 Huang G M, Tarn T J, Clark J W. On the controllability of quantummechanical systems. J Math Phys, 1983, 24: 2608-2618

9 Ong C K, Huang G M, Tarn T J, et al. Invertibility of quantummechanical control systems. Math Sys Theory, 1984, 17: 335-350

10 Clark J W, Ong C K, Tarn T J, et al. Quantum nondemolition filters. Math Sys Theory, 1985, 18: 33-55

11 Belavkin V P, Grishanin B A. Optimum measurement of quantum variables. Prob Info Transmission, 1972, 8: 259-265

12 Belavkin V P, Grishanin B A. Optimum estimation in quantum channels by the generalized heisenberg inequality method. Prob Info Transmission, 1972, 9: 209-215

13 Belavkin V P. Optimal quantum filtration of markovian signals. Prob Info Transmission, 1978, 7: 1-14

14 Rice S A, Zhao M. Optical Control of Molecular Dynamics. New York: John Wiley, 2000

15 Shapiro M, Brumer P. Principles of the Quantum Control of Molecular Processes. Hoboken: Wiley-Interscience, 2003

16 Bandrauk A D, Delfour M C, Le Bris C. Quantum control: Mathematical and numerical challenges. In: CRM Proceedings \& Lecture notes, American Mathematical Society, Providence, 2003

17 Warren W S, Rabitz H, Dahleh M. Coherent control of quantum dynamics: The dream is alive. Science, 1993, 259: 1581-1588

18 Rabitz H, de Vivie-Riedle R, Motzkus M, et al. Whither the future of controlling quantum pheonomena? Science, 2000, 288: 824-828

19 Brumer P, Shapiro M. Control of unimolecular reactions using coherent light. Chem Phys Lett, 1986, 126: 541-546

20 Shapiro M, Brumer P. Laser control of product quantum state populations in unimolecular reactions. J Chem Phys, 1986, 84: 4103-4104

21 Tannor D J, Rice S A. Control of selectivity of chemical reaction via control of wave packet evolution. J Chem Phys, 1985, 83: 5013-5018

22 Tannor D J, Kosloff R, Rice S A. Coherent pulse sequence induced control of selectivity of reactions: Exact quantum mechanical calculations. J Chem Phys, 1986, 85: 5805-5820

23 Zewail A H. Femtochemistry: Atomic-scale dynamics of the chemical bond. J Phys Chem A, 2000, 104: 5660-5694

24 Bonacic-Koutechy V, Mitric R. Theoretical exploration of ultrafast dynamics in atomic clusters: Analysis and control. Chem Rev, 2005 , 105: 11-65

25 Judson R S, Rabitz H. Teaching lasers to control molecules. Phys Rev Lett, 1992, 68: 1500

26 Dantus M, Lozovoy V. Experimental coherent laser control of physicochemical processes. Chem Rev, 2004, 104: 1813-1859

27 Brif C, Chakrabarti R, Rabitz H. Control of quantum phenomena: Past, present, and future. New J Phys, 2010, 12: 075008

28 Bucksbaum P, Eisenstein R. Controlling the Quantum World: The Science of Atoms, Molecules, and Photons. Washington, D.C.: The National Academies Press, 2007

29 D'Alessandro D. Introduction to Quantum Control and Dynamics. Boca Raton: Chapman \& Hall/CRC, 2008

30 Wiseman H. Quantum Measurement and Control. Cambridge: Cambridge University Press, 2010

31 Dong D, Petersen I. Quantum control theory and applications: A survey. IET Contr Theo Appl, 2010, 4: 2651-2671

32 Cao W C, Liu X S, Bai H B, et al. Bang-bang control suppression of 
amplitude damping in a three-level atom. Sci China Ser G-Phys Mech Astron, 2008, 51: 29-37

33 Qi B. On the quantum master equation under feedback control. Sci China Ser F-Inf Sci, 2009, 52: 2133-2139

34 Cheng D Z. Advances in automation and control research in China. Sci China Ser F-Inf Sci, 2009, 52: 1954-1963

35 Yuan Q, Li J H. Controlled quantum state transfer via parity measurement. Sci China Ser G-Phys Mech Astron, 2009, 52: 1203-1207

36 Yan S L. Control of chaos in an external-cavity multi-quantum-well laser subjected to dual-wedges and optical dual-feedback. Chin Sci Bull, 2009, 54: 1158-1163

37 Wei H R, Di Y M, Yan W. Synthesis of some three-qubit gates and their implementation in a three spins system coupled with ising interaction. Sci China Phys Mech Astron, 2010, 53: 664-671

38 André A, Bajscy M, Childress L, et al. Quantum control of light using coherent atomic memory. In: Atomic Physics 19: XIX International Conference on Atomic Physics 2004. AIP Conf Proc, 2005, 770: 291300

39 Bartels R A, Murnane M M, Kapteyn H C, et al. Learning from learning algorithms: Application to attosecond dynamics of high-harmonic generation. Phys Rev A, 2004, 70: 043404

40 Isidori A. Nonlinear Control Systems. New York: Springer-Verlag, 1995

41 Gardiner C W, Collett M J. Input and output in damped quantum systems: Quantum stochastic differential equations and the master equation. Phys Rev A, 1985, 31: 3761-3774

42 Gough J E, James M R, Nurdin H I. Quantum master equation and filter for systems driven by fields in a single photon state. In: Joint 50th IEEE Conference on Decision and Control (CDC) and European Control Conference (ECC), 2011

43 Lieb E. The stability of matter. Rev Mod Phys, 1976, 48: 553-569

44 Combescure M. The quantum stability problem for some class of timedependent hamiltonians. Ann Phys, 1988, 185: 86-110

45 Casati G, Chirikov B, Guarneri I, et al. Dynamic stability of quantum chaotic motion in a hydrogen-atom. Phys Rev Lett, 1986, 56: 2437 2440

46 D'Helon C, James M R. Stability, gain, and robustness in quantum feedback networks. Phys Rev A, 2006, 73: 053803

47 Lan C, Tarn T J, Chi Q S, et al. Analytic controllability of timedependent quantum control systems. J Math Phys, 2005, 46: 052102

48 Wu R B, Tarn T J, Li C W. Smooth controllability of infinite-dimensional quantum-mechanical systems. Phys Rev A, 2006, 73: 012719

49 Schirmer S G, Fu H, Solomon A I. Complete controllability of quantum systems. Phys Rev A, 2001, 63: 063410
50 Bloch A M, Brockett R W, Rangan C. Finite controllability of infinite-dimensional quantum systems. IEEE Trans Auto Contr, 2010, 55: 1797-1805

51 Wu R B, Hsieh M, Rabitz H. Role of controllability in optimizing quantum dynamics. Phys Rev A, 2011, 83: 062306

52 Chelkowski S, Bandrauk A D. Raman chirped adiabatic passage: A new method for selective excitation of high vibrational states. J Raman Spectr, 1997, 28: 459-466

53 Bergmann K, Theuer H, Shore B W. Coherent population transfer among quantum states of atoms and molecules. Rev Mod Phys, 1998 , 70: 1003-1025

54 Schirmer S, Greentree A, Ramakrishna V, et al. Constructive control of quantum systems using factorization of unitary operators. J Phys A, 2002, 35: 8315

55 Palao J, Kosloff R. Quantum computing by an optimal control algorithm for unitary transformations. Phys Rev Lett, 2002, 89: 188301

56 Zhang J, Li C W, Wu R B, et al. Maximal suppression of decoherence in markovian quantum systems. J Phys A, 2005, 38: 6587

57 D'Alessandro D. Optimal contro of two level quantum systems. IEEE Trans Auto Contr, 2001, 46: 866-876

58 Khaneja N, Brockett R, Glaser S J. Time optimal control in spin systems. Phys Rev A, 2001, 63: 032308

59 Zhu W S, Rabitz H. A rapid monotonically convergent iteration algorithm for quantum optimal control over the expectation value of a positive definite operator. J Chem Phys, 1998, 109: 385-391

60 Khaneja N, Reiss T, Kehlet C, et al. Optimal control of coupled spin dynamics: Design of nmr pulse sequences by gradient ascent algorithms. J Magn Reson, 2005, 172: 296-305

61 Rothman A, Ho T S, Rabitz H. Quantum observable homotopy tracking control. J Chem Phys, 2005, 123: 134104

62 Rothman A, Ho T S, Rabitz H. Observable-preserving control of quantum dynamics over a family of related systems. Phys Rev A, 2005, 72: 023416

63 Shir O M, Beltrani V, Bäck T, et al. On the diversity of multiple optimal controls for quantum systems. J Phys B, 2008, 41: 074021

64 Rabitz H, Hsieh M, Rosenthal C. Quantum optimally controlled transition landscapes. Science, 2004, 303: 1998-2001

65 Wu R, Rabitz H, Hsieh M. Characterization of the critical submanifolds in quantum ensemble control landscapes. J Phys A, 2008, 41: 015006

66 Rabitz H, Hsieh M, Rosenthal C. The landscape for optimal control of quantum-mechanical unitary transformations. Phys Rev A, 2005, 72: 52337

67 Wu R, Pechen A, Rabitz H, et al. Control landscapes for observable preparation with open quantum systems. J Math Phys, 2008, 49: 022108

Open Access This article is distributed under the terms of the Creative Commons Attribution License which permits any use, distribution, and reproduction in any medium, provided the original author(s) and source are credited. 